IASSNS-HEP-95/73

hep-th/9509118

\title{
SELF-TRAPPING OF THE DILATON
}

\author{
Rainer Dick \\ School of Natural Sciences, Institute for Advanced Study \\ Olden Lane, Princeton, NJ 08540, USA \\ and \\ Department of Physics, University of Munich \\ Theresienstr. 37, 80333 Munich, Germany
}

\begin{abstract}
The dilaton in three dimensions does not roll. Witten's conjecture that duality between theories in three and four dimensions solves the cosmological constant problem thus may also solve the dilaton problem in string theory.
\end{abstract}


Recently Witten pointed out that theories with Bogomol'nyi saturated solitons may be related to theories in higher dimensions in a Kaluza-Klein type framework [1, 2]. This idea can be motivated from classical duality considerations which generically imply a trading between solitons and particles in dual theories. Given the soliton-particle correspondence and the infinite tower of equidistant solitonic excitations it seems very natural to expect that theories with Bogomol'nyi saturated solitons are related to compactified theories in higher dimensions. In this framework the particular case of dualities between supersymmetric theories in $2+1$ dimensions and non-supersymmetric theories in $3+1$ dimensions deserves special attention, since the four-dimensional theory might inherit the vanishing of the cosmological constant from the corresponding three-dimensional theory [ [2, 7]. A recent discussion of the supersymmetric abelian Higgs model in $2+1$ dimensions coupled to supergravity confirmed this picture by showing that the soliton spectrum in this theory is not supersymmetric [8].

However, one might expect difficulties with this scenario due to the logarithmic potential in $2+1$ dimensions. In this note I would like to point out that the infrared singularity of the dilaton in three dimensions actually helps to solve the dilaton problem in string theory, since it implies a self-trapping of the dilaton: In $2+1$ dimensions fermions and adjoint scalars provide sources for the dilaton which differ in sign from the dilaton sources provided by the gauge fields which arise from the four-dimensional metric and four-dimensional gluons. Finite energy then implies that any local dilaton source has to be compensated by another dilaton source somewhere else, whence the dilaton vanishes asymptotically. In particular, the dilaton may fluctuate, but it does not roll. Hence, in the three-dimensional scenario string theory predicts fluctuations of low-energy couplings, but not a monotonic evolution.

In order to make this observation quantitative, I will also take a four-dimensional point of view like in [7] and discuss the action of the $(D-1)$-dimensional dilaton arising through a Kaluza-Klein parametrization of Einstein-Yang-Mills theory in $D$ dimensions. The Kaluza-Klein Ansatz (see e.g. [9])

$$
G_{M N}=\Phi^{-\frac{1}{D-2}}\left(\begin{array}{cc}
g_{\mu \nu}+\Phi a_{\mu} a_{\nu} & \Phi a_{\mu} \\
\Phi a_{\nu} & \Phi
\end{array}\right)
$$

yields in the gravitational sector (for the zero modes of $\partial_{D-1}$ and up to surface terms)

$$
\sqrt{-G} G^{M N} R_{M N}=\sqrt{-g}\left[g^{\mu \nu} R_{\mu \nu}-\frac{D-3}{4(D-2)} g^{\mu \nu} \partial_{\mu} \ln \Phi \cdot \partial_{\nu} \ln \Phi-\frac{\Phi}{4} f_{\mu \nu} f^{\mu \nu}\right]
$$

\footnotetext{
${ }^{1}$ Supersymmetry as a solution to the cosmological constant problem has been discussed in [3]. A very useful review and critical discussion of several attempts to solve the problem can be found in 沺. Early references on supergravity in $2+1$ dimensions are [5, 6].
} 
while reduction of the Yang-Mills field $A_{M}^{\alpha} \rightarrow A_{\mu}^{\alpha}, A^{\alpha}$ yields

$$
\begin{gathered}
-\frac{1}{4} \sqrt{-G} F_{M N}^{\alpha} F_{\alpha}^{M N}=-\frac{1}{4} \sqrt{-g} \Phi^{\frac{1}{D-2}}\left[B_{\mu \nu}^{\alpha} B_{\alpha}^{\mu \nu}+\frac{2}{\Phi} g^{\mu \nu} D_{\mu} A^{\alpha} \cdot D_{\nu} A_{\alpha}\right] \\
B_{\mu \nu}=F_{\mu \nu}+a_{\mu} D_{\nu} A-a_{\nu} D_{\mu} A
\end{gathered}
$$

We discuss reduction of the fermion terms for even $D$, since the case of odd $D$ is simpler, and we are mainly interested in the case $D=4$. If $\gamma_{\mu}$ is a basis of Dirac matrices in $D-1$ dimensions, a convenient basis of Dirac matrices in $D$ dimensions is given by

$$
\begin{gathered}
\Gamma_{0}=\left(\begin{array}{ll}
0 & 1 \\
1 & 0
\end{array}\right) \\
\Gamma_{j}=\left(\begin{array}{cc}
0 & -\gamma_{0} \gamma_{j} \\
\gamma_{0} \gamma_{j} & 0
\end{array}\right) \\
\Gamma_{D-1}=\left(\begin{array}{cc}
0 & \gamma_{0} \\
-\gamma_{0} & 0
\end{array}\right)
\end{gathered}
$$

The reduction of fermions yields both inequivalent representations of the Clifford algebra in $D-1$ dimensions. In a gauge $E^{\mu-1}=0$ for the $D$-bein and with a Kaluza-Klein Ansatz

$$
\Psi=\Phi^{\frac{1}{4(D-2)}}\left(\begin{array}{c}
\psi_{+} \\
\psi_{-}
\end{array}\right)
$$

the result reads

$$
\begin{gathered}
\sqrt{-G} \bar{\Psi}\left[E_{A}^{M} \Gamma^{A}\left(i \partial_{M}+i \Omega_{M}+q A_{M}\right)-M\right] \Psi= \\
=\sqrt{-g}\left[\bar{\psi}_{+} e^{\mu}{ }_{a} \gamma_{+}^{a}\left(i \partial_{\mu}+i \omega_{+\mu}+q V_{\mu}\right) \psi_{+}+\bar{\psi}_{-} e^{\mu}{ }_{a} \gamma_{-}^{a}\left(i \partial_{\mu}+i \omega_{-\mu}+q V_{\mu}\right) \psi_{-}\right] \\
-q \sqrt{-g} \Phi^{-\frac{1}{2}}\left(\bar{\psi}_{+} A \psi_{+}+\bar{\psi}_{-} A \psi_{-}\right)+M \sqrt{-g} \Phi^{-\frac{1}{2(D-2)}}\left(\psi_{+}^{+} \psi_{-}+\psi_{-}^{+} \psi_{+}\right) \\
-\frac{i}{8} \sqrt{-g} \Phi^{\frac{1}{2}} f_{a b}\left(\bar{\psi}_{+} \gamma_{+}^{a b} \psi_{+}+\bar{\psi}_{-} \gamma_{-}^{a b} \psi_{-}\right)
\end{gathered}
$$

with $\Omega$ and $\omega_{ \pm}$denoting the canonical spin connections, $\gamma_{ \pm}^{0}= \pm \gamma^{0}, \gamma_{ \pm}^{j}=\gamma^{j}$, and

$$
\begin{gathered}
V_{\mu}=A_{\mu}-a_{\mu} A \\
V_{\mu \nu}=B_{\mu \nu}-A f_{\mu \nu}
\end{gathered}
$$

In order to evaluate the effect of the infrared divergence of the electrostatic potential in $2+1$ dimensions, we consider the energy of a static configuration with the 
fermions in stationary orbits, and in gauge $A_{0}=a_{0}=0$ (complying with $E^{\mu}{ }_{D-1}=0$, since we employ diffeomorphisms which are constant along $\left.x^{D-1}\right)$ :

$$
\begin{gathered}
\frac{\mathcal{H}}{\sqrt{-g}}=\frac{D-3}{4(D-2)} g^{i j} \partial_{i} \ln \Phi \cdot \partial_{j} \ln \Phi+\frac{\Phi}{4} f_{i j} f^{i j}+\frac{1}{4} \Phi^{\frac{1}{D-2}}(V+A f)_{i j}^{\alpha}(V+A f)_{\alpha}^{i j} \\
+\frac{1}{2} \Phi^{-\frac{D-3}{D-2}} g^{i j} D_{i} A^{\alpha} \cdot D_{j} A_{\alpha}+q \Phi^{-\frac{1}{2}}\left(\bar{\psi}_{+} A \psi_{+}+\bar{\psi}_{-} A \psi_{-}\right)-M \Phi^{-\frac{1}{2(D-2)}}\left(\psi_{+}^{+} \psi_{-}+\psi_{-}^{+} \psi_{+}\right) \\
-\bar{\psi}_{+} e^{j}{ }_{a} \gamma_{+}^{a}\left(i \partial_{j}+i \omega_{+j}+q V_{j}\right) \psi_{+}-\bar{\psi}_{-} e^{j}{ }_{a} \gamma_{-}^{a}\left(i \partial_{j}+i \omega_{-j}+q V_{j}\right) \psi_{-} \\
+\frac{i}{8} \Phi^{\frac{1}{2}} f_{a b}\left(\bar{\psi}_{+} \gamma_{+}^{a b} \psi_{+}+\bar{\psi}_{-} \gamma_{-}^{a b} \psi_{-}\right)
\end{gathered}
$$

which tells us that the $U(1)$ gauge field and the Yang-Mills fields yield positive sources for the dilaton, while the kinetic term of $A$ provides a negative contribution. There is some ambiguity with regard to the contribution due to the fermions. However, on-shell the fermion contribution adds up to a positive term. Therefore, generically the fermions will contribute negative sources to the dilaton.

This appearance of positive and negative source terms for the dilaton apparently works for any value of $D$. Of course, the negative terms are absent in the tendimensional formulation of superstring theory since eleven-dimensional supergravity does not contain elementary fermions and Yang-Mills fields.

However, the case $D=4$ is peculiar due to the logarithmic IR divergence of the electrostatic potential in $2+1$ dimensions. In a linear approximation the dilaton $\ln \Phi$ behaves like a massless scalar field coupled to external sources, whence finiteness of energy requires a vanishing dilaton charge:

$$
\begin{gathered}
\int d^{2} \mathbf{x}\left(\frac{1}{4} f_{i j} f^{i j}+\frac{1}{8}(V+A f)_{i j}^{\alpha}(V+A f)_{\alpha}^{i j}-\frac{1}{4} g^{i j} D_{i} A^{\alpha} \cdot D_{j} A_{\alpha}\right. \\
\left.-\frac{1}{2} q\left(\bar{\psi}_{+} A \psi_{+}+\bar{\psi}_{-} A \psi_{-}\right)+\frac{1}{4} M\left(\psi_{+}^{+} \psi_{-}+\psi_{-}^{+} \psi_{+}\right)+\frac{i}{16} f_{a b}\left(\bar{\psi}_{+} \gamma_{+}^{a b} \psi_{+}+\bar{\psi}_{-} \gamma_{-}^{a b} \psi_{-}\right)\right)=0
\end{gathered}
$$

This property is similar to charge neutrality of the Coulomb gas in two dimensions and can be derived from conformal invariance of the partition function or independence of the arbitrary length scale entering the definition of the electrostatic potential. It can also be inferred from the fact that the retarded potential $\frac{\Theta(t-r)}{2 \pi \sqrt{t^{2}-r^{2}}}$ yields a finite dilaton for static configurations only if the sources add up to zero.

The self-trapping mechanism encoded in (6) is superficially stable with respect to quantum effects, since calculation of the 1-loop effective potential in the linear approximation and in presence of an ultraviolet cutoff $\Lambda$ yields a $\Lambda^{3} \cosh (\ln \Phi)$-type potential of the dilaton. However, consideration of an effective potential in a nonrenormalizable theory is rather speculative, and it is clear that like in the four-dimensional theory 
with gravity a proper treatment of quantum effects would imply genuine stringy considerations.

The physics behind (6) is more transparent than in the case of the Coulomb gas, since particles and antiparticles contribute in the same way to the dilaton: If a gauge boson excites a dilaton field the divergence of the resulting energy density implies pair production of adjoint scalars and fermions to restore an asymptotically vanishing dilaton. Clearly, $M$ suppresses the production of fermions relative to adjoint scalars. Stated in another way: The adjoint scalar and light fermions screen the dilaton charge of the gauge bosons.

The observed stability of the dilaton in this framework is not in contradiction with unwinding of the fourth dimension, since in the parametrization (1) the length $L$ of the range of $x^{D-1}$ determines the radius of the internal dimension rather than the dimensionless dilaton $\Phi$. Rescaling $x^{D-1}$ to an angular variable through the substitution $\Phi \rightarrow L^{4} \Phi$ shows immediately that the reasoning above only implies stability of an arbitrary large internal dimension against low energy excitations. Unwinding should arise as a nonperturbative string effect rather than a property of a low energy effective field theory with time-dependent couplings.

On the other hand, there is also another possibility, which I would prefer: There is no unwinding of the fourth dimension, since it was never curled up. Anything involved in the previous investigation was a lowest order Kaluza-Klein parametrization, which only requires translational invariance along $x^{3}$, but there is no truncation of $x^{3}$ to a finite interval. This also implies absence of an energy gap: We were only looking at the lowest edge of a continuum, approximated in the dual supersymmetric formulation by saturated solitons. The content of the three-dimensional string scenario is both remarkable and simple: The low energy degrees of freedom and the supersymmetry which we expect to inherit from string theory motivate considerations of three-dimensional effective theories, but this strictly does not imply compactification of seven dimensions. It is pretty clear that the simple Kaluza-Klein parametrization (1) employed here represents at best a crude approximation to lowest order string theory, and we have to think more thorougly about the geometry and meaning of the 3-manifolds involved in the infrared limit of string theory. It is a natural expectation that the role of the 3-manifolds will find an explanation within the holographic theory of space-time [10, 11], as has been pointed out already by Vafa, cf. [2].

The self-trapping of the dilaton explained above clearly does not depend on the particular embedding of $2+1$ dimensions. It thus turns out that the prospects to get rid of a rolling dilaton through the three-dimensional picture proposed by Witten are as excellent as the prospects to get rid of the cosmological constant. This scenario might open up an unexpected and interesting window to string phenomenology once we develop a better understanding of the role and the dynamics of 3-manifolds in four-dimensional quantum gravity, and Witten's proposal clearly deserves further 
study.

Acknowledgement: I would like to thank E. Witten for discussions and for hospitality at the Institute for Advanced Study. This work was supported by a grant from the DFG.

\section{References}

[1] E. Witten, Nucl. Phys. B443 (1995) 85, hep-th/9503124.

[2] E. Witten, Strong coupling and the cosmological constant, IASSNS-HEP-95/51, hep-th/9506101.

[3] B. Zumino, Nucl. Phys. B89 (1975) 535.

[4] S. Weinberg, Rev. Mod. Phys. 61 (1989) 1.

[5] P.S. Howe and R.W. Tucker, J. Phys. A10 (1977) 455; J. Math. Phys. 19 (1978) 869.

[6] S. Deser and J.H. Kay, Phys. Lett. 120B (1983) 97.

[7] E. Witten, Some comments on string dynamics, IASSNS-HEP-95/63, hep th/9507121.

[8] K. Becker, M. Becker and A. Strominger, Phys. Rev. D51 (1995) 6603, hep th/9502107.

[9] T. Appelquist and A. Chodos, Phys. Rev. D28 (1983) 772.

[10] L. Susskind, Phys. Rev. D49 (1994) 6606, hep-th/9308139; The world as a hologram, hep-th/9409089.

[11] G. 't Hooft, Dimensional reduction in quantum gravity, in Salamfest 1993, pp. 284-296, hep-th/9310026. 